\title{
On-site early-warning system for Bishkek (Kyrgyzstan)
}

\author{
Dino Bindi ${ }^{1}{ }^{\star}$, Tobias Boxberger $^{1}$, Sagynbek Orunbaev $^{2}$, Marco Pilz $^{1}$, Jacek Stankiewicz ${ }^{3}$, \\ Massimiliano Pittore ${ }^{1}$, Iunio Iervolino ${ }^{4}$, Enrico Ellguth ${ }^{5}$, Stefano Parolai ${ }^{1}$
}

\author{
${ }^{1}$ Helmholtz Center Potsdam - German Research Center for Geosciences, Potsdam, Germany \\ ${ }^{2}$ Central Asian Institute for Applied Geosciences, Bishkek, Kyrgyzstan \\ ${ }^{3}$ European Center for Geodynamics and Seismology, Walferdange, Luxembourg \\ ${ }^{4}$ University of Naples Federico II, Naples, Italy \\ ${ }^{5}$ GEMPA GmbH, Potsdam, Germany
}

\author{
Article history \\ Received September 18, 2014; accepted January 7, 2015. \\ Subject classification: \\ Seismology, Earthquake early warning, Central Asia.
}

\begin{abstract}
In this work, the development of an on-site early warning system for Bishkek (Kyrgyzstan) is outlined. Several low cost sensors equipped with MEMS accelerometers are installed in eight buildings distributed within the urban area. The different sensing units communicate each other via wireless links and the seismic data are streamed in real-time to the data center using internet. Since each single sensing unit has computing capabilities, software for data processing can be installed to perform decentralized actions. In particular, each sensing unit can perform event detection task and run software for on-site early warning. If a description for the vulnerability of the building is uploaded in the sensing unit, this piece of information can be exploited to introduce the expected probability of damage in the early-warning protocol customized for a specific structure.
\end{abstract}

\section{Introduction}

Nowadays, early warning systems are envisioned as an important component of risk management and mitigation procedures in regions exposed to various natural hazards. Examples are the earthquake early warning (EEW) systems currently in operation in countries like Japan [Doi 2011], Mexico [Espinosa-Aranda et al. 2011], and Taiwan [Hsiao et al. 2009] or the tsunami early warning system running in the Pacific and Indian Ocean [Lauterjung et al. 2010]. Recent earthquakes, such as the March 20, 2012, Mw 7.4 Ometepec earthquake (Mexico) showed the effectiveness of EEW through the issue of an alert to the population, and actions such as an orderly shutdown of the subway system [e.g., Yamasaki 2012]. In Japan, the EEW is in operation since 2007, with many alerts issued to a population having a high level of awareness and preparedness, which are fundamental ingredients of any successful early warning system. The recent
2011 M 9 Tohoku earthquake shook large areas of Japan with JMA intensity 7 (largest value in that scale) and produced ground acceleration exceeding $1 \mathrm{~g}$ even at distances larger than $300 \mathrm{~km}$ from the epicenter. Although with an underestimated magnitude of 7.2, the early warning was issued to public about $8.6 \mathrm{~s}$ after the first trigger, and before the S-wave arrival [Hoshiba and Ozaki 2014]. The warnings were, therefore, issued rapidly but underestimating the effect of the earthquake due to its large size, with a rupture time much longer than the $S$ P time observed at those triggered stations which mainly control the outcome of the early warning system. Since the usefulness of the EEW was anyway confirmed, those responsible for the Japanese EEW started the development and implementation of methodologies to cope both with size of earthquakes large enough to lead to saturation in the magnitude scale, and with false alarms issued during the aftershock sequence [Hoshiba and Ozaki 2014]. Among the many elements composing an EEW system, the network configuration determines which approach is applied to determine the early warning information.

Two main configurations are generally implemented, namely the regional (or network based) and on-site (or single station based) approaches. In the former, a network of stations is deployed close to the source area in a way to detect the occurrence of an earthquake early enough to characterize its location and magnitude before the destructive $S$-waves hit the target location(s). The warning- (or lead-) time is computed as the difference between the arrival time of $S$-wave in the target site and the time at which the event was detected. The network generally computes the location and magni- 
tude of the event, and uses empirical ground motion prediction equations to provide probabilistic estimates of the ground motion at the target site.

In the on-site approach the stations are installed close to the target. The early warning procedure is then generally based on using parameters estimated over few seconds of recorded P-waves to predict the ground motion for $\mathrm{S}$-waves, avoiding the location of earthquake and its magnitude estimation. Combination of the two approaches has been also proposed [e.g., Zollo et al. 2010].

The on-site early warning benefited from the rapid technological developments of the last decades. Low cost sensing units with embedded computational power and wireless communication have been continuously developed since ' 80 s [Lynch and Kenneth 2006], allowing the deployment of dense urban strong motion networks, [e.g., Fleming et al. 2009, Fisher et al. 2012]. The possibility of the sensing units to query the data allows for decentralized operations and the communication link between several stations can be exploited to implement coincidence trigger algorithms that can limit the number of false-positive detections. Furthermore, simplified structural models can be uploaded in the system to estimate, in real-time and on-site, the behavior of nearby buildings. When the units are installed for building monitoring, the recordings can be used to update the vulnerability model, following the performance based approach [Iervolino 2011] accounting for time-dependent hazard [Iervolino et al. 2014].

Considering the advantages of early warning systems, many other countries are developing and evaluating them, like USA [Böse et al. 2014], Romania [Ionescu et al. 2007], Italy [Zollo et al. 2014]. Besides the above mentioned regions, where efforts for developing EEW are ongoing for a long time, many other regions of the world exposed to high seismic hazard and risk are not monitored by strong motion networks and EEW system are not developed. An example of such region is Central Asia, including the countries of Kazakhstan, Kyrgyzstan, Tajikistan, Uzbekistan, and Turkmenistan. If, on one hand, several previous studies quantified the hazard of this area as one of the highest in the world [e.g., Ulomov and the GSHAP Region 7 Working Group 1999, Bindi et al. 2012], on the other hand almost no strong motion monitoring is performed in this area after the end of the Soviet Union. The aim of this article is to present the results of a first attempt to create an urban strong motion network in Bishkek (Kyrgyzstan) for early warning, rapid response and building monitoring purposes.

We first briefly recall the technical characteristics of the sensing unit [Fleming et al. 2009], as well as the software for on-line early warning installed inside each unit. Then, we outline the installation in different buildings of the town and we conclude with the envisaged installation of a regional EEW to be coupled with the on-site ones [Pittore et al. 2014].

\section{Sosewin wireless sensing unit}

One of the first wireless sensing units (WSUs) for structural health monitoring was proposed by Straser and Kiremidjian [1998]. Since that first application, WSUs have been improved following the technological advances [e.g., Lynch and Kenneth 2006]. In particular, the possibility of equipping these sensing units with microcontrollers and computational units has allowed users to optimize the WSU performance and to run decentralized damage analysis programs [e.g., Lynch et al. 2004]. Within the framework of the EU-FP6 SAFER (Seismic Early Warning for Europe; http:/ / www.saferproject.net/) and German EDIM (Earthquake Disaster Information system for Marmara region, Turkey; http: / / www.cedim.de/EDIM.php) projects, the Helmholtz Centre Potsdam GFZ, German Research Centre for Geosciences, in collaboration with the Department of Informatics of the Humboldt University-Berlin, developed a WSU called SOSEWIN, Self-Organizing Seismic Early Warning Information Network [Fleming et al. 2009, Picozzi et al. 2010, Fischer et al. 2012]. Several aims drove the development of the GFZ-WSU, among which the main are:

- each seismological sensing unit or sensing node (SN) is made of low-cost "off-the-shelf" components;

- SOSEWIN is a decentralized, self-organizing $a d$ hoc wireless mesh network (WMN);

- each SN undertakes its own, on-site seismological data processing, preliminary analysis, archiving, and communication of data, as well as the issuing of early warning messages;

- the early-warning decision-making is carried out within the WMN of sensing units, taking advantage of their communication capability and the design of suitable alarming processes; thus, the alarming itself can be done both inside the network (i.e., flooding the alarm to every node) and outside it (i.e., routing the alarm to the nearest gateway node, and then toward some external administrative center);

- its self-organizing capability allows it to adapt continuously to changing circustances; e.g., the addition/removal/malfunctioning of nodes, interference in communications due to local (and possibly time-varying) phenomena, loss of sections of the network following an earthquake, etc.

These characteristics made SOSEWIN suitable for a dense, self-organizing and decentralized seismic monitoring network for both structural health monitoring 
(i.e., working in near-real-time) and also for earthquake early warning (i.e., working in real-time) applications

The main components of the SOSEWIN sensing unit are shown in Figure 1. The communication of seismic data and information amongst the GFZ-WSU within a network is based on the routing concept. The term routing refers to the procedure of selecting within a network the paths along which data can be sent from a source to a sink. Routing activities within a wireless network are complicated by the fact that all nodes act contemporarily as sources, sinks and routers of data. GFZ-WSUs rely on the OLSR (Optimized Link State Routing) protocols as the routing strategy, and are designed to form, as described above, a self-organizing $a d-$ hoc WMN. The OLSR that the GFZ-WSU employs is a proactive routing protocol, where every node has a map of the complete network topology, allowing data to be immediately sent along the optimal path towards the users or gateways (i.e., other nodes that will then transmit the information to, for example, an early warning center). This leads to each node having a routing table that describes the most efficient way to reach every other node. It makes use of advanced metrics; i.e., measurement methods, for the evaluation of a multi-hop path within the network.

The WSU of SOSEWIN was used in many applications, both for structural monitoring [Fleming et al. 2009, Oth and Picozzi 2012], and for geophysical surveys based on surface-wave data analysis [e.g., Picozzi et al. 2010]. In particular, SOSEWIN installations were used to monitor a building in Italy during the 2009 L'Aquila sequence [Picozzi et al. 2011]. Soon after the April 6, 2009, Mw 6.3 main shock, the German Earthquake Task Force was involved in the installation of a temporary seismological network to support their Italian colleagues. In particular, under the request and coordination of the Italian Government Department of Civil Protection (or DPC), SOSEWIN accelerometric sensing units were installed within selected strategic infrastructures, both damaged and undamaged, for the recording of aftershocks and the real-time determination of characteristic building parameters. The city hall building of Navelli village, located about 30 $\mathrm{km}$ south of L'Aquila, was instrumented to monitor the damage generated by the main shock [Mucciarelli et al. 2010]. Among other outcomes, an interferometric approach applied to the SOSEWIN recordings allowed to follow the changes in the fundamental frequency of resonance of the building during the sequence [Picozzi et al. 2011].

In conclusion, the self-organizing character allows GFZ-WSU to automatically adapt to changes in the network configuration, and guarantees the functionality

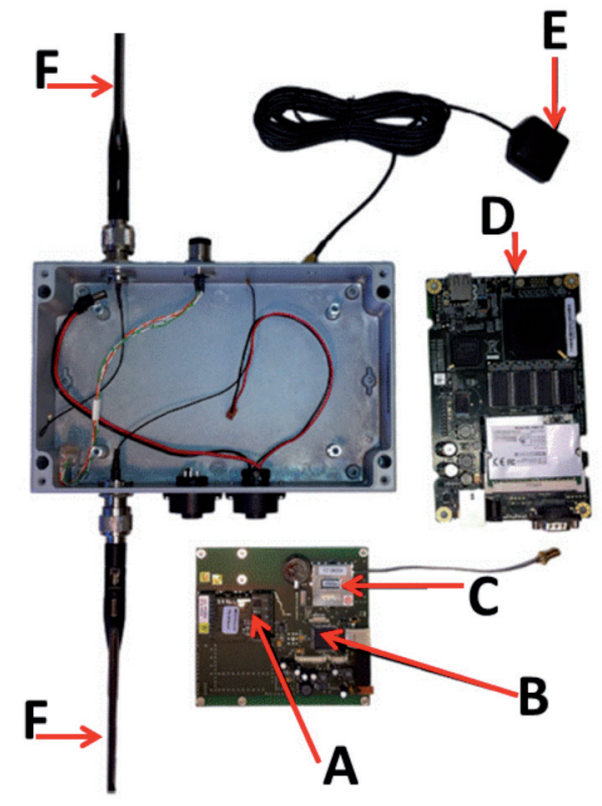

Figure 1. Main components of the SOSEWIN sensing unit. A: MEMS sensor; B: microcontroller; C: GPS receiver; D: Main board; E: GPS antennas; F: WLAN antennas.

of the network, even when some of the sensing units malfunction, or cannot be seen by the external user. Moreover, the possibility of deploying dense networks allows for the realization of on-site earthquake early warning/rapid response deployments within mega cities. On the other hand, the possibility of analyzing the data at the sensing unit level and broadcasting the results not only to a data management center, but also to the other nodes of the network, allows one to set up decentralized protocols for structural health monitoring and early warning.

\section{Software for decentralized analysis}

The computational power of each WSU allows each node to query and process the recorded data and to disseminate the results through the network. In particular, a data processing and analysis module for onsite early warning is installed in each node, performing the following actions:

- the real-time data streams are filtered using a 4 th order recursive Butterworth bandpass filter (typically $0.075-25 \mathrm{~Hz}$ );

- the filtered data are integrated to both velocity and displacement using the recursive scheme of Kanamori et al. [1999];

- an event detection algorithm based on short-term average/long-term average (STA/LTA) is applied; when the ratio exceeds a predefined threshold, a trigger condition is declared

- once triggered, the node computes several parameters over a P-wave window with pre-defined duration (typically 3 seconds); the most relevant parameters 
are the maximum acceleration (PGA), velocity (PGV), displacement (PGD) and the predominant period $\left(\tau_{c}\right)$;

- the parameters measured over the early P-wave arrivals are used to predict some parameters for the $S$ wave, by using empirical relations. In particular, the PGD measured over the early vertical P-wave is used to estimate the PGV for the horizontal S-wave by applying the Zollo et al. [2010] relationship, calibrated considering earthquakes occurred in Taiwan, Japan, Italy and valid for distances up to $60 \mathrm{~km}$;

- a combination of parameters (e.g. PGD and $\tau_{c}$ ) can also be exploited to estimate the magnitude [e.g., Kuyuk and Allen 2013].

The procedure implemented in each sensing unit is sketched in Figure 2. The predicted ground motion for $\mathrm{S}$-waves is the input information for an alert scheme based on thresholds in ground acceleration or seismic intensity values [Pittore et al. 2014]. The exact definition of the alert scheme requires a strong interaction with end-users (e.g., local Ministry of Emergency Situation).

The software has been implemented using the SeiscomP3 libraries (www.seiscomp3.org), compiled in each sensing units. The CAPS (Common Acquisition Protocol Server, http:/ / www.gempa.de/caps/) is used to manage the communication of the data streams in real-time, directed toward the other sensing units, when present, and to a server located in the data management center. The advantage of the CAPS server over the more common Seedlink protocol (http://www.seiscomp3. org/wiki/doc/applications/seedlink) is the possibility of handling multi-parametric streams in one unified protocol, allowing to efficiently transfer information from different sensors, such as seismic sensors, images, or hydro-meteo sensors. A messaging system is also implemented and managed through SeiscomP3, allowing the sensing unit, after the occurrence of a trigger, to report about the status of the measurements and the relevant prediction.

The sensing unit can also upload a physical description of the vulnerability of the hosting building, moving toward the direction of implementing the performance based EEW [Iervolino 2011]. If fragility curves suitable for the hosting structure are available, the system can predict the probabilities of different damage levels using the ground motion predicted for S-wave from the P-wave parameters recorded at the ground level (Figure 2). Note that, in principle, these fragility curves may also be updated, accounting for post-event damage conditions of the structure, for the early warning during a potential next event (e.g., an aftershock), which is no more hitting an intact structure.
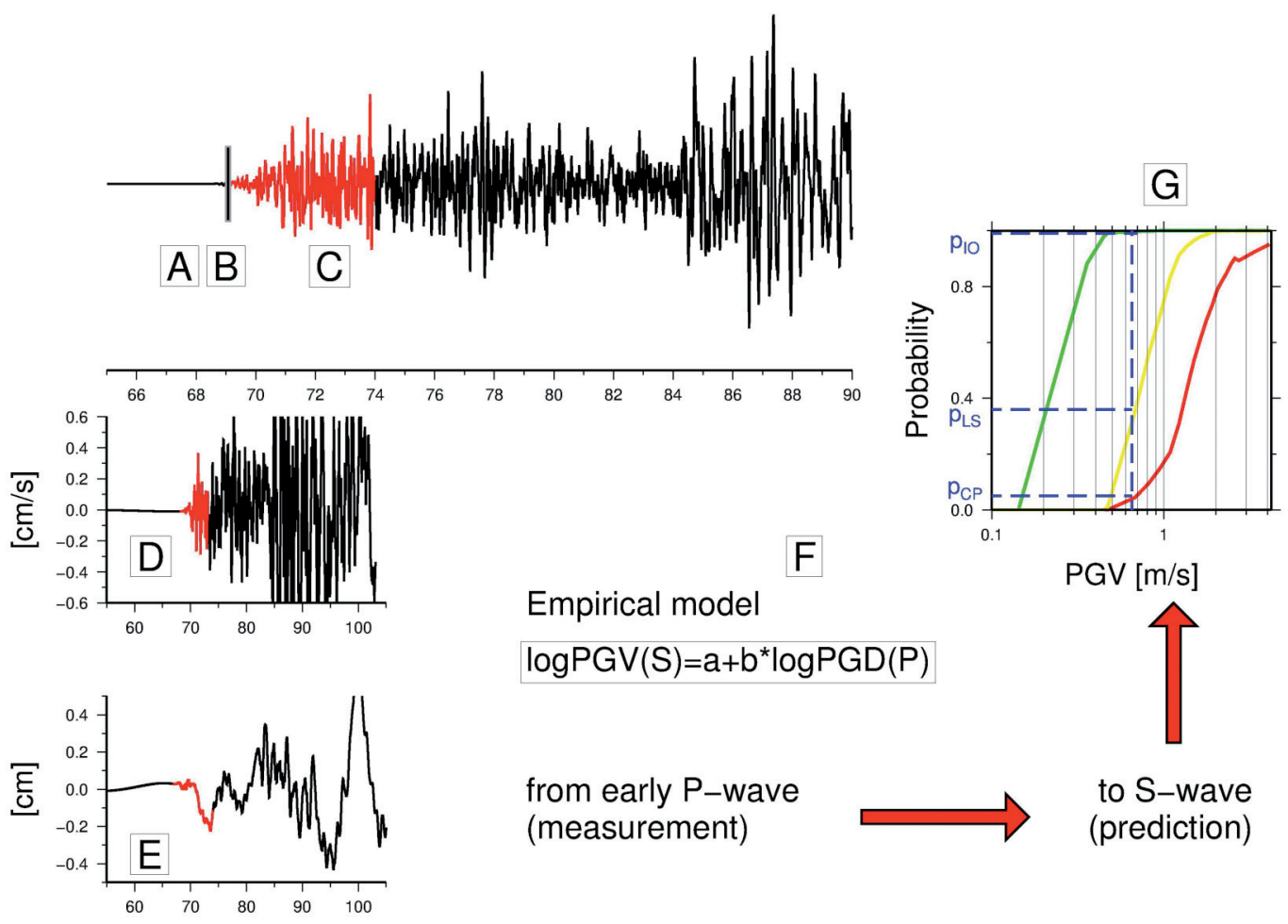

(measurement)

to S-wave

(prediction)

Figure 2. Sketch of the single station on-site EEW algorithm. A: real-time data processing; B: trigger based on short-time over long-time average; C: estimation of strong motion parameters over 3 seconds after triggering; D: estimation of parameters over velocity integrated signal; E:estimation of parameters over displacement integrated parameters; F: prediction of PGV for S-waves using the PGD over P-waves and using empirical relations; G: when available, the fragility curves of the building are used to compute the probabilities of damage using both the PGV predicted from P-waves and observed. See text for details. 


\section{Installation in Bishkek}

Several previous studies showed that Bishkek, capital of Kyrgyzstan (Central Asia) is exposed to high seismic hazard and risk [e.g., Ulomov and the GSHAP Region 7 Working Group 1999, Erdik et al. 2005, Bindi et al. 2011, Bindi et al. 2012, Wieland et al. 2012, Pittore and Wieland 2013]. Among the actions for mitigating the seismic risk in Bishkek, a regional early warning system is under development [Picozzi et al. 2012, Stankiewicz et al. 2013, Pittore et al. 2014]. Since the regional early warning system is conceived for earthquakes generated at some tens of kilometers from Bishkek while Bishkek is also located in close proximity to the Issyk-Ata fault, an on-site system is under development to complete the regional one. It is worth noting that, while the on-site system can produce a useful lead time for distances at which the regional one is blind [e.g. Satriano et al. 2011], an earthquake occurring over the Issyk-Ata fault could be close enough to Bishkek to show a travel time difference between $\mathrm{S}$ - and P-waves smaller than 3 seconds. In such a case, only threshold based approaches can be exploited.

The aim of the on-site network is twofold: on the one hand, to realize a prototype for on-site EEW as described in the previous sections; on the other hand, to continuously monitor buildings in Bishkek to improve the knowledge about the seismic response of the former Soviet time building stock. With these objectives in mind, eight buildings have been selected and equipped with sensing units installed at different floors, for a total
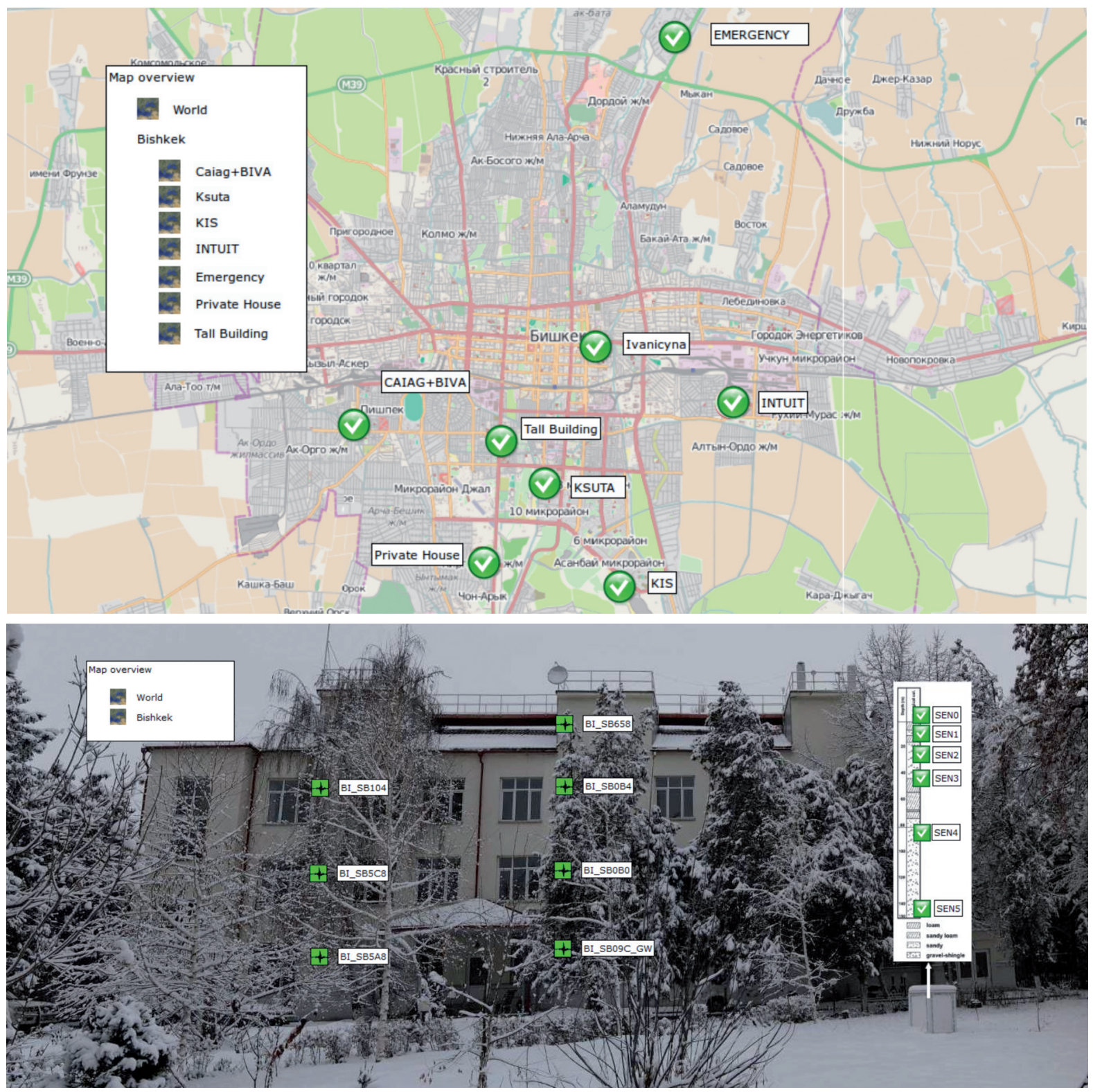

Figure 3. Top: location of the 8 buildings monitored in Bishkek. The communication link for the building tagged with the red symbol is under development. Bottom: example of distribution of sensing units in one building (CAIAG institute). In the yard of the institute a vertical array equipped with 6 accelerometers is also operating [Parolai et al. 2013]. 

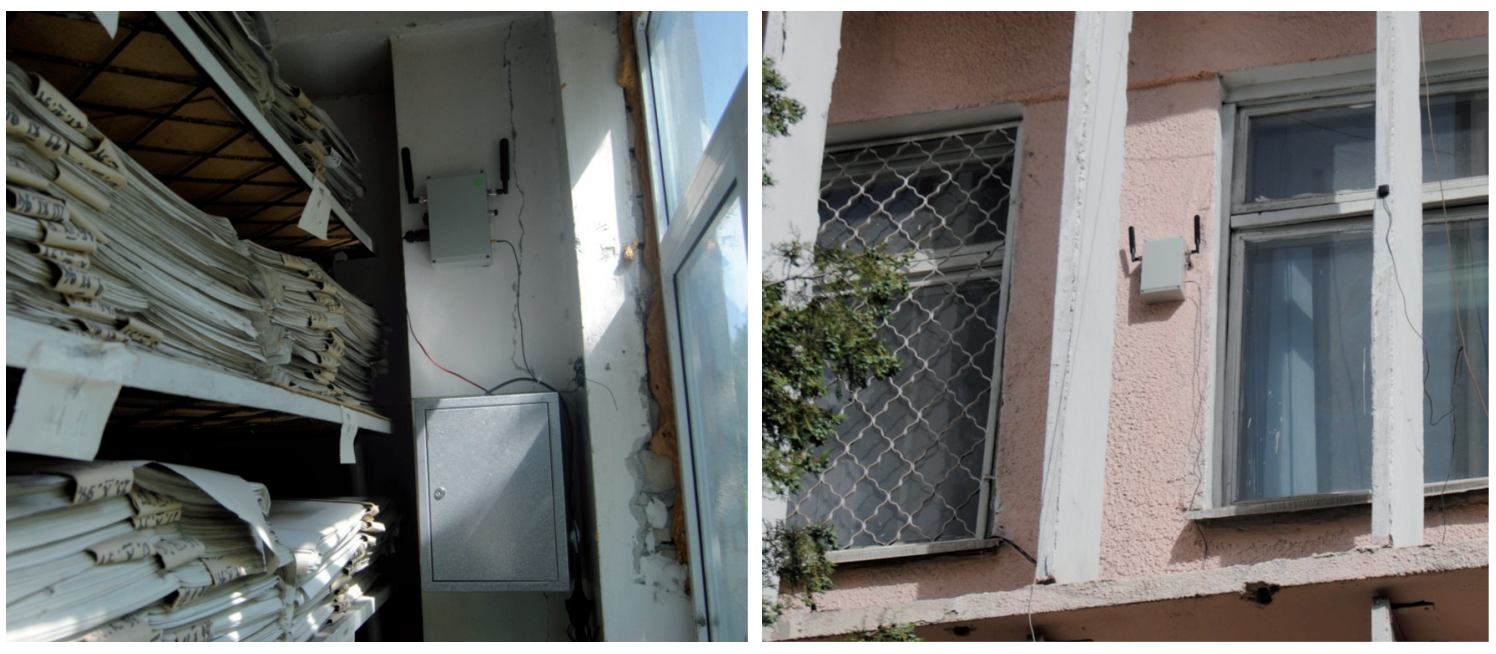

Figure 4. Examples of sensing unit installed in the Institute of Seismology (left) and KSUTA University (right).

of thirty-two instruments. From each building, the data are transmitted to the data center located in CAIAG (Central Asian Institute for Applied Geosciences), where a vertical array (6 strong motion sensors down to a depth of $150 \mathrm{~m}$ ) is also operating in real-time to study the effects of seismic wave propagation in the uppermost layers [Parolai et al. 2013]. The location of the monitored building is shown in Figure 3, while examples of installation are shown in Figure 4. Among the selected buildings, five are public buildings (i.e., two universities, two research institutes, one building belonging to the Ministry of Emergency Situation) and three residential buildings, representative of different construction types. The recorded data are transmitted from each building to the CAIAG institute internet connection. A buffer communication link based on long range WIFI communication is currently under test.

\section{Conclusions}

Earthquake early warning systems can provide information, which in turn can be used to mitigate the consequences of an earthquake in real-time. The technological advances of the last thirty years allow the installation of sensing units with computational power to run a decentralized early warning system. Moreover, new developments in EEW are moving towards at performance-based systems and the embedding of the EEW into rapid assessment of risk. Bishkek is a test site in Central Asia for developing both a regional and an on-site EEW system. Although designed to react to different situations, these two systems will work together, each providing useful constraints to the other. For example, the regional EEW system implements modules for the rapid dissemination of loss maps in Bishkek [Pittore et al. 2014]. The availability of not only the ground motion recorded in Bishkek by the on-site system but also an estimate of the probabilities of damage when a fragility description is uploaded in the sensing units, can allow for the addition of valuable information to the one already avaialble from the regional system. The on-site EEW in Bishkek is currently under development and testing, with the major open issues related to the stability of the communication link and the power supply.

Acknowledgements. The activities described in this article have been founded by the EMCA-Earthquake Model Central Asia. The quality of the paper was enhanced by the comments of Adrien Oth.

\section{References}

Bindi, D., M. Mayfield, S. Parolai, S. Tyagunov, U. Begaliev, K. Abdrakhmatov, B. Moldobekov and J. Zschau (2011). Towards an improved seismic risk scenario for Bishkek, Kyrgyz republic, Soil Dyn. Earthq. Eng., 31, 521-525.

Bindi, D., K. Abdrakhmatov, S. Parolai, M. Mucciarelli, G. Grünthal, A. Ischuk, N. Mikhailova and J. Zschau (2012). Seismic hazard assessment in Central Asia: outcomes from a site approach, Soil Dyn. Earthq. Eng., 37, 84-91.

Böse, M., R. Allen, H. Brown, G. Cua, M. Fischer, E. Hauksson, T. Heaton, M. Hellweg, M. Liukis, D. Neuhauser, P. Maechling and CISN EEW Group (2014). CISN ShakeAlert - An Earthquake Early Warning Demonstration System for California, In: F. Wenzel and J. Zschau (eds.), Early Warning for Geological Disasters - Scientific Methods and Current Practice, Springer, Berlin-Heidelberg-New York, ISBN 978-3-642-12232-3.

Doi, K. (2011). The operation and performance of earthquake early warnings by the Japan Meteorological Agency, Soil Dyn. Earthq. Eng., 31, 119-126.

Erdik, M., T. Rashidov, E. Safak and A. Turdukulov (2005). Assessment of seismic risk in Tashkent, Uzbekistan and Bishkek, Kyrgyz republic, Soil Dyn. Earthq. 
Eng., 25, 473-486.

Espinosa-Aranda, J.M., A. Cuellar, F.H. Rodriguez, B. Frontana, G. Ibarrola, R. Islas and A. Garcia (2011). The seismic alert system of Mexico (SASMEX): progress and its current applications, Soil Dyn. Earthq. Eng., 31, 154-162.

Fischer, J., J.-P. Redlich, J. Zschau et al. (2012). A wireless mesh sensing network for early warning, J. Netw. Comput. Appl., 35, 538-547; doi:10.1016/j.jnca.2011. 07.016

Fleming, K., M. Picozzi, C. Milkereit, F. Kühnlenz, B. Lichtblau, J. Fischer, C. Zulfikar and O. Ozel (2009). The Self-organizing Seismic Early Warning Information Network (SOSEWIN), Seismol. Res. Lett., 80 (5), 755-771.

Hoshiba, M., and T. Ozaki (2014). Earthquake Early Warning and Tsunami Warning of the Japan Meteorological Agency, and their performance in the 2011 off the Pacific Coast of Tohoku earthquake (Mw 9.0), In: F. Wenzel and J. Zschau (eds.), Early Warning for Geological Disasters - Scientific Methods and Current Practice, Springer, Berlin-Heidelberg-New York, ISBN 978-3-642-12232-3.

Hsiao, N.-C., Y.-M. Wu, T.-C. Shin, L. Zhao and T.-L. Teng (2009). Development of earthquake early warning system in Taiwan, Geophys. Res. Lett., 36, L00B02; doi:10.1029/2008GL036596.

Iervolino I. (2011). Performance-based earthquake early warning, Soil Dyn. Earthq. Eng., 31, 209-222.

Iervolino, I., M. Giorgio and E. Chioccarelli (2014). Closed-form aftershock reliability of damage-cumulating elastic-perfectly-plastic systems, Earthq. Eng. Struct. D., 43, 613-625.

Ionescu, C., M. Böse, F. Wenzel, A. Marmureanu, A. Grigore and G. Marmureanu (2007). An Early Warning System for Deep Vrancea (Romania) Earthquakes, In: P. Gasparini, G. Manfredi and J. Zschau (eds.), Earthquake Early Warning Systems, Springer, Berlin-Heidelberg.

Kanamori, H., P. Maechling and E. Hauksson (1999). Continuous monitoring of ground-motion parameters, B. Seismol. Soc. Am., 89, 331-316.

Kuyuk, H.S., and R.M. Allen (2013). A global approach to provide magnitude estimates for earthquake early warning alerts, Geophys. Res. Lett., 40, 6329-6333; doi:10.1002/2013GL058580.

Lauteriung, J., U. Münch and A. Rudloff (2010). The challenge of installing a tsunami early warning system in the vicinity of the Sunda Arc, Indonesia, Nat. Hazards Earth Syst. Sci., 10, 641-646.

Lynch, J.P., A. Sundararajan, H. Sohn et al. (2004) Embedding actuation functionalities in a wireless structural health monitoring system, Proc, 1st Int, Work.
Adv. Smart Mater. Smart Struct. Technol.

Lynch, J.P., and J.L. Kenneth (2006) A Summary Review of Wireless Sensors and Sensor Networks for Structural Health Monitoring, Shock Vib. Dig., 38, 91128; doi:10.1177/0583102406061499.

Mucciarelli, M., M. Bianca, R. Ditommaso, M.R. Gallipoli, A. Masi, C. Milkereit, S. Parolai, M. Picozzi and M. Vona (2010). Far field damage on RC buildings: the case study of Navelli during the L'Aquila (Italy) seismic sequence, 2009, B. Earthq. Eng., 9, 263-283; doi:10.1007/s10518-010-9201-y.

Oth, A., and M. Picozzi (2012). Structural Health Monitoring using wireless technologies: an ambient vibration test on the Adolphe Bridge, Luxembourg City, Advances in Civil Engineering, 17 pp.; doi:10. $1155 / 2012 / 876174$.

Parolai, S., D. Bindi, S. Ullah, S. Orunbaev, S. Usupaev, B. Moldobekov and H. Echtler (2013). The Bishkek vertical array (BIVA): acquiring strong motion data in Kyrgyzstan and first results, J. Seismol., 17 (2), 707719.

Picozzi, M., C. Milkereit, S. Parolai, K.-H. Jaecke, I. Veit, J. Fischer and J. Zschau (2010). GFZ Wireless Seismic Array (GFZ-WISE), a Wireless Mesh Network of Seismic Sensors: New Perspectives for Seismic Noise Array Investigations and Site Monitoring; Sensors, 10, 3280-3304; doi:10.3390/s100403280.

Picozzi, M., S. Parolai, M. Mucciarelli, C. Milkereit, D. Bindi, R. Ditommaso, M. Vona, M.R. Gallipoli and J. Zschau (2011). Interferometric analysis of strong ground motion for structural health monitoring: the example of the L'Aquila, Italy, seismic sequence of 2009, B. Seismol. Soc. Am., 101, 635-651.

Picozzi, M., D. Bindi, M. Pittore, K. Kieling and S. Parolai (2012). Real-time risk assessment in seismic early warning and rapid response: a feasibility study in Bishkek (Kyrgyzstan), J. Seismol.; doi:10.1007/s1095 0-012-9332-5.

Pittore, M., and M. Wieland (2013). Toward a rapid probabilistic seismic vulnerability assessment using satellite and ground-based remote sensing, Nat. Hazards, 68, 115-145.

Pittore, M., D. Bindi, J. Stankiewicz, A. Oth, M. Wieland, T. Boxberger and S. Parolai (2014). Towards a Lossdriven Earthquake Early Warning and Rapid Response system for Kyrgyzstan (Central Asia), Seismol. Res. Lett., 85 (6), 1328-1340; doi:10.1785 / 0220140106.

Satriano, C., Y-M. Wu, A. Zollo and H. Kanamori (2011). Earthquake early warning: concepts, methods and physical grounds, Soil Dyn. Earthq. Eng., 31, 106118.

Stankiewicz, J., D. Bindi, A. Oth and S. Parolai (2013). Designing efficient earthquake early warning sys- 
tems: case study of Almaty, Kazakhstan, J. Seismol., 17, 1125-1137; doi:10.1007/s10950-013-9381-4.

Straser, E.G., and A.S. Kiremidjian (1998). A Modular, Wireless Damage Monitoring System for Structures, Report no. 128, John A. Blume Earthquake Engineering Center, Stanford University, Palo Alto, CA, USA.

Ulomov, VI, and the GSHAP Region 7 Working Group (1999). Seismic hazard of Northern Eurasia, Annali di Geofisica, 42 (6), 1023-1038.

Yamasaki, E. (2012). What We Can Learn From Japan's Early Earthquake Warning System, Momentum 1, vol. 1, article 2, published by ScholarlyCommons; http:// repository.upenn.edu/momentum/vol1/iss $1 / 2$.

Wieland, M., M. Pittore, S. Parolai, J. Zschau, B. Moldobekov and U. Begaliev (2012). Estimating building inventory for rapid seismic vulnerability assessment: towards an integrated approach based on multisource imaging, Soil Dyn. Earthq. Eng., 36, 70-83.

Zollo, A., O. Amoroso, M. Lancieri, Y.-M. Wu and H. Kanamori (2010). A threshold-based earthquake early warning using dense accelerometer networks, Geophys. J. Int., 183, 963-974.

Zollo, A., S. Colombelli, L. Elia, E. Emolo, G. Festa, G. Iannacone, C. Martino and P. Gasparini (2014). An integrated regional and on-site Earthquake Early Warning system for southern Italy: concepts, methodologies and performances, In: F. Wenzel and J. Zschau (eds.), Early Warning for Geological Disasters - Scientific Methods and Current Practice, Springer, Berlin-Heidelberg-New York, ISBN 978-3642-12232-3.

${ }^{\star}$ Corresponding author: Dino Bindi,

Helmholtz Center Potsdam - German Research Center for Geosciences, Potsdam, Germany; email: bindi@gfz-potsdam.de. 